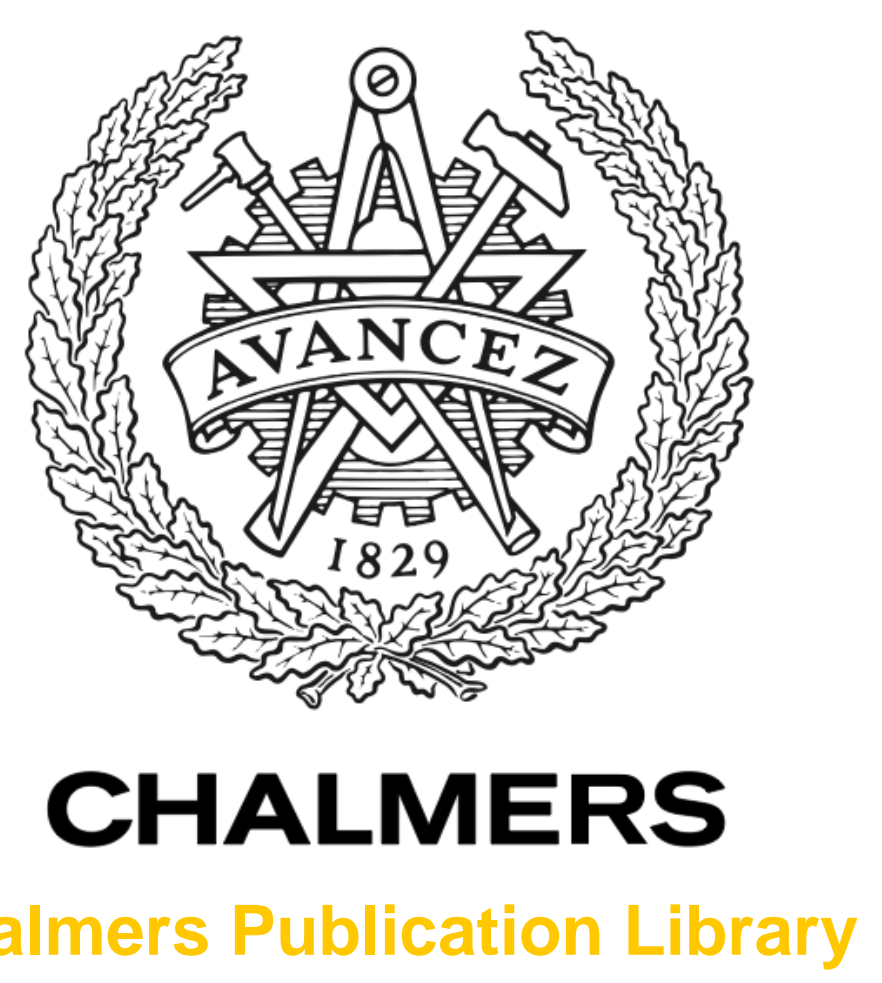

Challmers Publication Library

High-Power Broadly Tunable Electrooptic Frequency Comb Generator

This document has been downloaded from Chalmers Publication Library (CPL). It is the author's version of a work that was accepted for publication in:

leee Journal of Selected Topics in Quantum Electronics (ISSN: 1077-260X)

Citation for the published paper:

Metcalf, A. ; Torres Company, V. ; Leaird, D. (2013) "High-Power Broadly Tunable

Electrooptic Frequency Comb Generator". Ieee Journal of Selected Topics in Quantum

Electronics, vol. 19(6),

http://dx.doi.org/10.1109/jstqe.2013.2268384

Downloaded from: http://publications.lib.chalmers.se/publication/185746

Notice: Changes introduced as a result of publishing processes such as copy-editing and formatting may not be reflected in this document. For a definitive version of this work, please refer to the published source. Please note that access to the published version might require a subscription.

Chalmers Publication Library (CPL) offers the possibility of retrieving research publications produced at Chalmers University of Technology. It covers all types of publications: articles, dissertations, licentiate theses, masters theses, conference papers, reports etc. Since 2006 it is the official tool for Chalmers official publication statistics. To ensure that Chalmers research results are disseminated as widely as possible, an Open Access Policy has been adopted.

The CPL service is administrated and maintained by Chalmers Library. 


\title{
High-Power Broadly Tunable Electro-Optic Frequency Comb Generator
}

\author{
Andrew J. Metcalf, Student Member, IEEE, Victor Torres-Company, Daniel E. Leaird, Senior \\ Member, IEEE, and Andrew M. Weiner, Fellow, IEEE
}

\begin{abstract}
Broadband travelling-wave electro-optic modulators made of lithium niobate have reached a high level of technological maturity. They can provide simultaneously low $\mathrm{V} \pi$, sustain high power (both optical and RF) and yet provide low propagation loss. By combining together these features, we present a high-power handling, broadly tunable, electro-optic frequency comb generator. The device produces between 60-75 lines within -10 $\mathrm{dB}$ bandwidth over its full tuning range -from 6$18 \mathrm{GHz}-$ and can handle up to $1 \mathrm{~W}$ of optical input power. This optical frequency comb platform is very well suited for applications in RF photonics and optical communications that require independent $R F$ and optical tuning as well as highrepetition rates but moderate bandwidth.
\end{abstract}

Index Terms -Electrooptic modulators, Microwave photonics, Optical communication equipment, Ultrafast optics.

\section{INTRODUCTION}

$\mathrm{T}$ HE frequency comb laser has revolutionized the field of optical synthesis and metrology [1]. There are two important attributes that distinguish an optical frequency comb from any other multiwavelength laser source, i.e., high phase coherence across the whole optical bandwidth and the possibility to tune independently the repetition rate and frequency offset [2]. With the advent of selfreferenced mode-locked lasers [3,4], researchers can measure or synthesize absolute optical frequencies with a level of precision that was previously achieved only in very specialized laboratories. The accessibility to such an

A. J. Metcalf (e-mail: metcalfa@purdue.edu), D. E. Leaird (email: leaird@purdue.edu) and A. M. Weiner (e-mail: amw@purdue.edu) are at the School of Electrical and Computer Engineering, Purdue University, West Lafayette, IN 47906 USA.

V. Torres-Company is at the Microtechnology and Nanoscience department, Chalmers University of Technology, Gothenburg, Sweden (email: torresv@chalmers.se).

This work was supported by the Naval Postgraduate School under grant N00244-09-1-0068 under the National Security Science and Engineering Faculty Fellowship program. Any opinion, findings, and conclusions or recommendations expressed in this publication are those of the authors and do not necessarily reflect the views of the sponsors. unprecedented level of performance has triggered a non-stop expansion of applications based on precision metrology [5]. However, apart from one notable exception [6], mode-locked Ti:Sa or erbium-doped fiber lasers usually operate at repetition rate frequencies below $10 \mathrm{GHz}$. Notwithstanding, there are novel applications of frequency combs, relying upon the previously mentioned two features, which do not require selfreferencing but high repetition rates. We mention, for instance, optical communications [7-9], radiofrequency photonics [10-13], and optical arbitrary waveform generation [14]. For this type of applications, a more suitable platform is the socalled electro-optic frequency comb generator [15]. The general scheme of this type of comb consists of a continuous-wave (CW) laser sent to a set of electro-optic modulators driven by an external RF oscillator. The modulation introduces sidebands around the optical frequency defined by the input laser. Hence, the performance of this platform is dictated by the noise characteristics of the laser, oscillator, optical components, and microwave amplifiers. This solution combines robustness and simplicity at a few $\mathrm{nm}$ bandwidth (considering $\sim 10$ $\mathrm{GHz}$ oscillator and state-of-the-art opto-electronic and microwave components). Electro-optic comb generators may be categorized in resonant $[16,17]$ or single-pass [15,18-28]. The former set consists of a single electro-optic modulator (usually phase) placed in a cavity, so that multiple sidebands are generated by resonant interaction. With the use of a narrow-linewidth laser locked to one of the cavity resonances, very low timing jitter has been reported [29]. An obvious drawback of this configuration is the loss of flexibility in continuously tuning the optical frequency or repetition rate of the comb. The single-pass configuration consists of placing various modulators in tandem. Hence, broad bandwidth 
operation is achieved in a relatively simple configuration as the laser light travels through the modulators. The frequency of the $\mathrm{CW}$ laser can be freely chosen within the spectral bandwidth of the modulators and optical components, whereas the RF settings shall be picked in accordance to the desired electro-optic modulation attributes. Again, multiple solutions exist in the literature featuring e.g. compactness [21,22], low-power consumption [23], and spectral flatness [19-26]. Among all the different available solutions, probably the most balanced configuration in terms of simplicity, flatness and tuning flexibility consists of one electro-optic intensity modulator and one or more phase modulators [26-28].

In the above configuration, the intensity modulator (IM) is biased to provide a train of pseudo-square pulses with $\sim 50 \%$ duty cycle, whereas the phase modulators (PMs) yield the broadband spectrum. If the temporal signals are correctly aligned in time, the IM will carve the light only when the chirp from the PMs is mostly linear, hence providing a relatively flat spectrum [26]. This process can be interpreted in an elegant manner in terms of the space-time analogy [30,31], where the phase modulation is a time-lensing effect [32] that induces a strong time-to-frequency mapping [33]. The typical "rabbit -ears" at the outer part of the spectrum, caused in this type of frequency comb platform, are due to the deviation of the parabolic phase modulation (or time lens aberrations) within the intensity profile [34]. If needed, this effect can be suppressed by correcting the aberrations with the aid of higher harmonics from the RF oscillator [32] and/or by narrowing the IM pulses [25].

In absence of aberration correction, the setup gains substantially in RF tuning flexibility and the power variation in the comb lines remain well below $10 \mathrm{~dB}$. The main drawback with this configuration relates to the tradeoff between bandwidth and throughput as more PMs are added into the setup. In addition, as the accumulated modulation index increases, one can naturally get concerned about increase of timing jitter. In this contribution, we have optimized the design for such electro-optic intensity + phase comb generator using state-of-the-art commercially available electro-optic lithium niobate modulators. As a result, we built a standalone frequency comb delivering $>13 \mathrm{dBm}$ output power operating over a broad and tunable range of repetition rates (from 6$18 \mathrm{GHz}$ ). The comb outputs between 60-73 lines within a $-10 \mathrm{~dB}$ bandwidth, depending on the chosen frequency. We include experimental results highlighting the power stability and provide evidence of minimum degradation of the noise in the oscillator frequency throughout the microwaveoptical-microwave transfer. We anticipate that the high-power characteristics, stability, and flexible operation of this source will likely enable new applications in radio-frequency photonics and coherent communications, where the highperformance synthesis of large time-bandwidthproduct waveforms is highly beneficial.

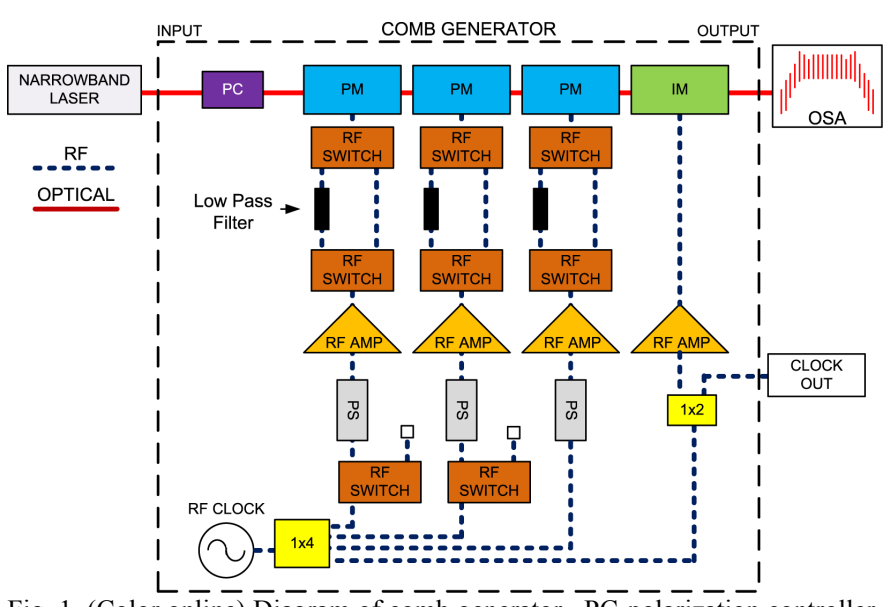

Fig. 1. (Color online) Diagram of comb generator. $\overline{\text { P }} \overline{\mathrm{C}}$-polarization controller, PM-phase modulator, IM-intensity modulator, RF AMP-radio frequency amplifier, PS-radio frequency phase shifter, $1 \times 2 \& 1 \times 4-$ radio frequency splitters, OSA -optical spectrum analyzer, RF SWITCH - radio frequency switches. The two RF switches before the phase shifters allow us to turn off RF power to their respective PM's. The group of six RF switches located after the RF AMPs switch between two RF paths, one filtered and one not.

\section{SETUP DESCRIPTION}

The layout of our comb generator is shown in Fig.1. A narrow-linewidth $(<10 \mathrm{kHz}) \mathrm{CW}$ fiber laser source is sent through a series of three electrooptic PMs and one electro-optic IM, all driven by the same tunable RF oscillator. Each modulator has a bandwidth of $20 \mathrm{GHz}$. The intensity modulator $\left(\mathrm{V}_{\pi} \sim 5.5 \mathrm{~V}\right.$ at $\left.10 \mathrm{GHz}\right)$ is carefully biased to carve out a flat-top pulse train from the CW laser. We then use the RF phase shifters (PS) to correctly align the cusp of the phase modulation from every PM with the peak of the flat top pulse. The optical bandwidth of the comb is proportional to the modulation index introduced by the cascade of PMs [26-28]. Each PM has $\mathrm{V}_{\pi} \sim 3.5 \mathrm{~V}$ at $10 \mathrm{GHz}$ and is 
capable to sustain $1 \mathrm{~W}$ of RF power, leading to a bandwidth of more than 20 lines within $-10 \mathrm{~dB}$ bandwidth per PM.

Usually the power handling limitation of regular modulators restricts the ability to increase the input laser power to get higher output comb power. Our design incorporates the first two PMs with highpower handling capabilities (up to $1 \mathrm{~W}$ ) while keeping low loss, and the comb delivers up to $13 \mathrm{dBm}$ output power at $10 \mathrm{GHz}$ repetition rate. To put this in perspective, our group's previous optoelectronic comb generator, detailed in [25], only output $-9 \mathrm{dBm}$ total power.

When dealing with multiple phase modulators, it is important to correctly align the chirp from each PM with the cusp of the pulses from the IM. This is not a straightforward task when all modulators are running at once. Usually, it requires disconnecting the PM's from the setup and aligning them one by one. To expedite the tuning process, we installed RF switches in-line with two of the PM's. The switches allow us to select each PM and align the phase of the amplified RF signal with the aid of the corresponding PS. After optimization, the effective modulation index corresponds to the summation of the modulation indices from each PM.

We noticed that when operating the comb at repetition rates of $11 \mathrm{GHz}$ and below, the second harmonic generated in the RF high-power amplifier falls within the bandwidth of the PM. This distorts the linear chirp and degrades the quality of the comb. We have been able to filter out these higher harmonics while still achieving operation over the full tunable range $(6-18 \mathrm{GHz})$. Our solution was to install a set of two RF switches after each RF amplifier. The first switch after the amplifier selects between two paths, one with a filter (K\&L 6L25012000/T26000) and one without (see Fig.1). The second switch simply recombines the chosen path with the input of the PM. This allows the user to select the operation mode between $6-11 \mathrm{GHz}$ (path with filter) or 11-18 GHz (path without filter).

\section{EXPERIMENTAL RESULTS}

\section{A. Tuning ability}

Our frequency comb generator is broadly tunable in repetition rate. By simply changing the RF clock frequency we can switch between different repetition rate combs spanning 6 to $18 \mathrm{GHz}$ (limited by the bandwidth of our RF-amplifiers). After setting the clock repetition rate, the comb can be reoptimized quickly, using the RF phase shifters to align the cusps of the phase modulation with the flat-top pulses. The key to our fast tuning is the ability to turn off the PM's, thus allowing us to align them individually. Our process is as follows. First, we use the RF switches to turn off RF power to two of the PM's, leaving only 1 PM and the IM receiving RF power. Then while monitoring the output spectrum via an OSA, we use the PS in-line with PM1 to obtain a symmetric comb spectrum. When symmetry is achieved we switch on RF power to a second $\mathrm{PM}$ and repeat the process optimizing again for a symmetric comb. After repeating the process for the 3rd PM the resultant tuning time to achieve a broadband flat-top comb is around one minute.
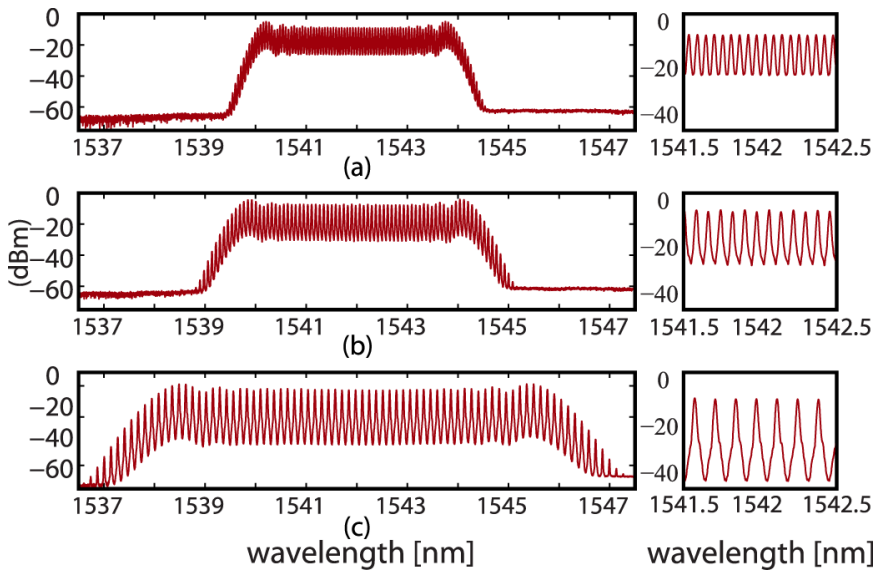

Fig. 2. (Color online) Comb spectra at output of comb generator with $1 \mathrm{~W}$ of optical input power. (a) $7 \mathrm{GHz}$ spectrum, 73 lines within $-10 \mathrm{~dB}$. (b) $10 \mathrm{GHz}$ spectrum, 65 lines within $-10 \mathrm{~dB}$. (c) $17 \mathrm{GHz}$ spectrum, 63 lines within $-10 \mathrm{~dB}$. Right column displays a $1 \mathrm{~nm}$ zoom of the corresponding combs on the left.

Spectral traces are shown in Fig. 2, for 7, 10, and $17 \mathrm{GHz}$, respectively. The limited extinction ratio of the comb lines at the lower repetition rates is attributed to the limited resolution $(0.01 \mathrm{~nm})$ of the optical spectrum analyzer (OSA). In these measurements the comb was operated with $1 \mathrm{~W}$ optical input power, which corresponds to the maximum sustainable power of the PMs. The output spectrum shows roughly -15 to $-17 \mathrm{~dB}$ loss for all three repetition rates, which is typical across the full tuning range. The majority of the loss can be attributed to the insertion loss of the optical 
components, $3 \mathrm{~dB}$ for each PM and $2 \mathrm{~dB}$ for the IM. This gives us a high maximum output power from 13 to $15 \mathrm{dBm}$.

\section{B. Pulse compression}

In order to test the coherence of our source, we generate a comb at $12 \mathrm{GHz}$, then compress it via line-by-line pulse shaping [14] using a commercial pulse shaper (Finisar Waveshaper 1000S). If the pulse is compressible to the bandwidth limited duration, we infer a high-degree of spectral phase stability. The autocorrelation trace is optimized in an iterative process by compensating for even-order terms of spectral phase up to eight. The autocorrelation trace shown in Fig. 3(a) corresponds to the one when the optimization procedure is finished. The measured AC trace is compared to the transform-limited case, which was calculated from the measured optical spectrum while assuming a flat spectral phase. The agreement between the curves is excellent, demonstrating high spectral-phase stability in the source.

Although we corrected up to 8th order with the shaper, the total phase applied was almost purely quadratic. For comparison, Fig. 3(b) shows the total programmed phase along with its best quadratic fit. Because the phase compensation is nearly quadratic, it allows for near band-limited pulse compression with dispersive fiber alone. Fig 3.(a) shows the autocorrelation trace if only quadratic compression was used.

It is also interesting to look at how the achievable pulse width varies across our tunable range. At our maximum repetition rate $(18 \mathrm{GHz})$, and keeping in mind that each PM adds about 20 additional lines, our maximum bandwidth is roughly $3 * 20 * 18=$ $1080 \mathrm{GHz}$, allowing for a sub-picosecond pulse. However, for our smallest repetition rate $(6 \mathrm{GHz})$ our total bandwidth is only $360 \mathrm{GHz}$. In order to achieve sub-picosecond pulses at this repetition rate it would require 8 total PM's, which would add additional loss.

\section{Power stability}

We measure the stability of our source by letting it free-run over the course of one hour, while monitoring the optical spectrum with the OSA.
Spectral traces were acquired at $0.01 \mathrm{~nm}$ resolution
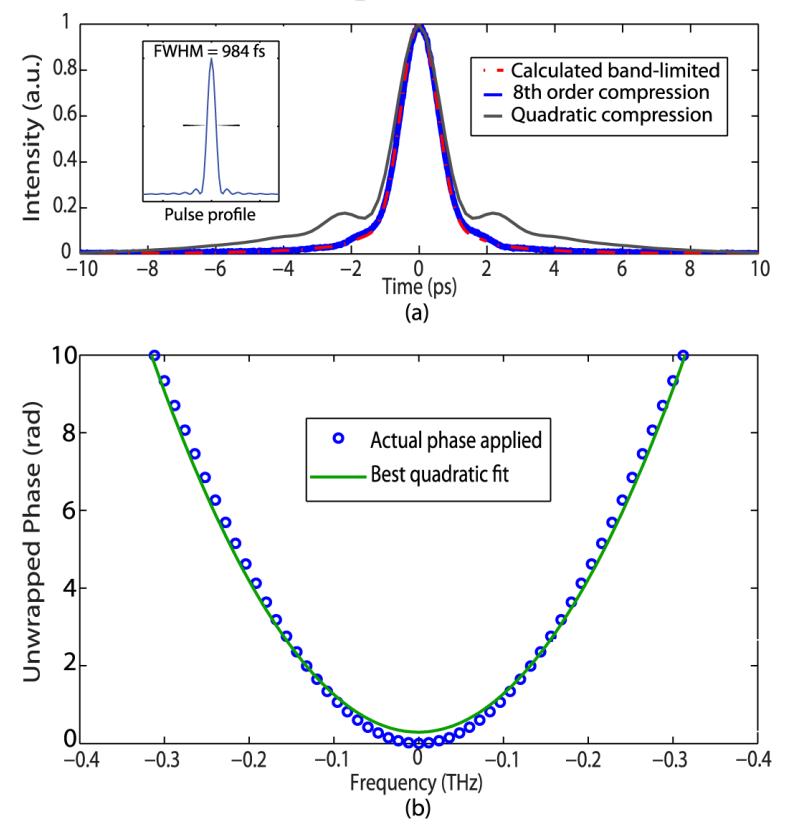

Fig. 3. (Color online) (a) Measured autocorrelation trace correcting up to 8th order with pulse shaper(blue). Expected autocorrelation calculated using measured spectrum and assuming flat phase (red). Expected autocorrelation calculated only using quadratic phase (grey). (Inset) Calculated band-limited pulse, FWHM 984 fs. (b) Total 8th order phase programmed with shaper for pulse compression (blue circles). Best quadratic fit to total phase applied (green).

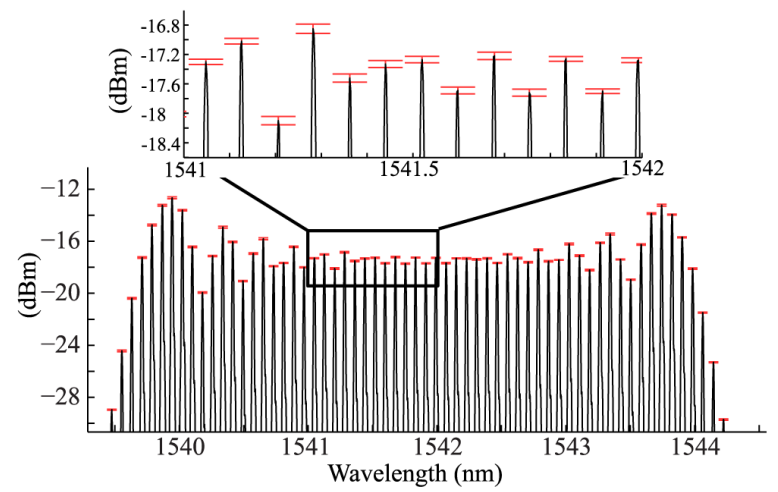

Fig. 4. (Color Online) Stability measurement of $10 \mathrm{GHz}$ comb over one hour, with measurement increments of 30 seconds. Black average comb, Red: standard deviation of comb amplitude.

every 30 seconds for the duration of the measurement. A spectral trace of the average comb over the duration of the measurement is given in Fig.4. The standard deviation of the measured fluctuations is shown in the error bars overlaying the average spectrum, showing a maximum standard deviation of $0.15 \mathrm{dBm}$. It should be noted that for time durations over a few hours the unstabilized DC bias will drift. This in turn will reshape the temporal profile of the IM, and slightly change the shape of the spectrum. However, within our one hour measurement, the absence of any 
active stabilization for the DC bias did not significantly affect the comb shape.

In addition, a separate measurement was taken in the time domain. The output of the comb after compression was converted to the electrical domain via a $22 \mathrm{GHz} \mathrm{PD}$, before being measured by a realtime scope (Tektronix DSA72004B). The waveform was sampled at $50 \mathrm{Gs} / \mathrm{s}$, with a trace recorded every four seconds for a total of one hour. Fig. 5, shows all of the sampled waveforms overlaid together. The maximum peak deviation is $\sim 12 \%$ of the overall pulse amplitude.

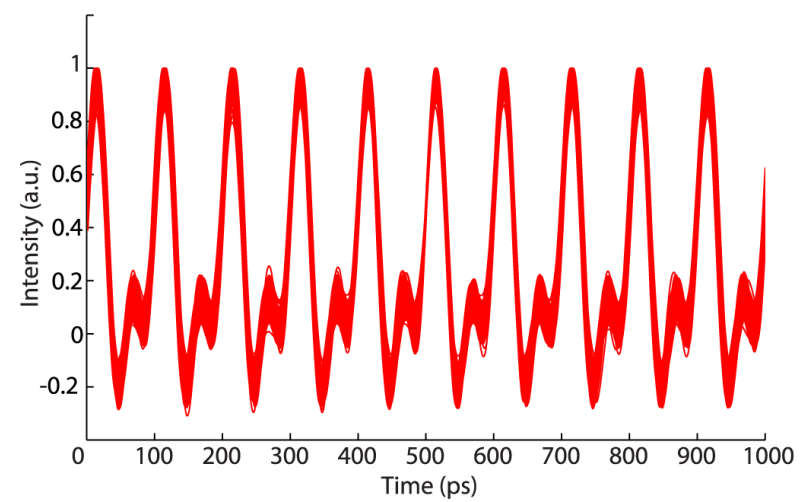

Fig. 5. (Color Online) Time domain stability measurement of $10 \mathrm{GHz}$ comb over one hour. Data was measured with a real-time scope sampling at 50Gs/s. A trace was recorded every 4 seconds over the course of an hour.

\section{Noise measurement}

Measurements comparing the single-sideband (SSB) noise RF spectrum of the RF oscillator and first tone of the photodetected intensity of our comb are displayed in Fig. 6. The measurements were carried out for three repetition rates, 7,10 , and 17 GHz. The pulses were first compressed with the aid of the pulse shaper to their near transform-limited duration. The spectral phase of the comb is compensated for to induce phase-to-intensity conversion from the three PM stage. This is done to make sure that the contribution to the SSB RF spectrum from the phase modulators is properly taken into account. The optical intensity is converted to the RF domain via a $22 \mathrm{GHz}$ photodiode. The RF signal was then amplified using two RF amplifiers (MITEQ AMF-6D and MiniCircuits ZVE-2W-183+) before being measured using the RF phase-noise utility of our electrical spectrum analyzer. For completeness, the noise measurement of the tunable RF oscillator (Hittite HMC-T2100) for each frequency, as well as the noise floor of the analyzer are also shown. We are able to see the phase noise of the comb matches almost exactly with that of the RF oscillator alone,

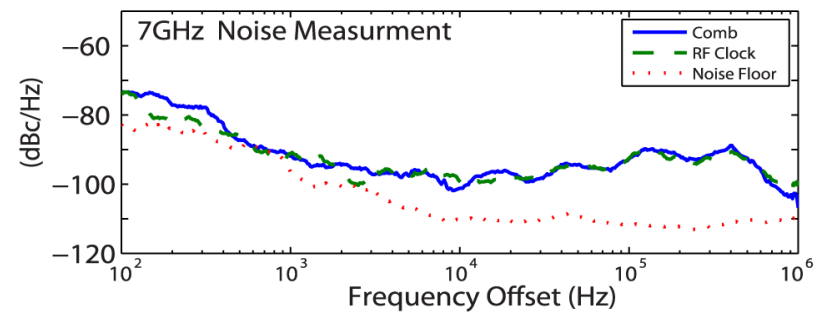

(a)

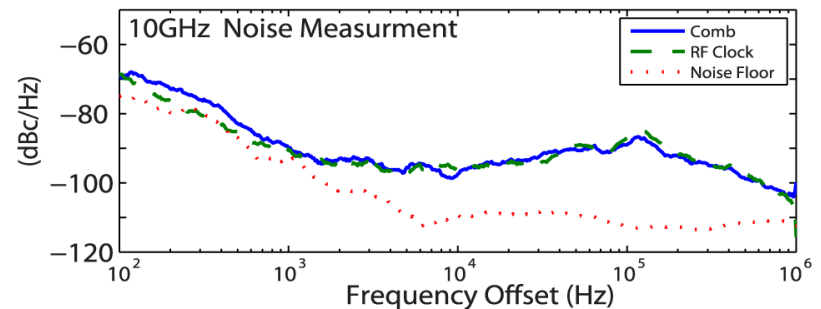

(b)

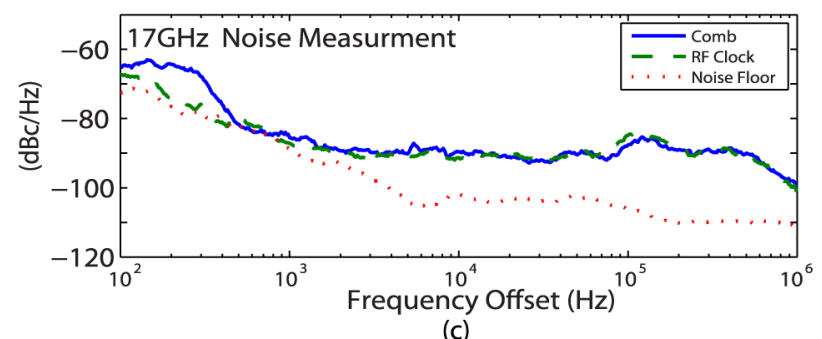

Fig. 6. (Color online) single-sideband RF spectrum-noise measurements for carrier signal of $7 \mathrm{GHz}$ (a), $10 \mathrm{GHz}$ (b), and $17 \mathrm{GHz}$ (c). Solid blue curveoutput from comb source, Green dashed curve- RF clock, Red-dotted curvenoise floor of analyzer.

indicating the comb generation process does not degrade the purity of the tone to a significant extent.

\section{CONCLUSION}

We have demonstrated a tunable, high power handling, frequency comb source made up of discrete electro-optic IM and PM's. The source proved to be extremely stable over a one hour time window, in which the RF clock source was free of any feedback. In addition we can conclude through the SSB-noise measurements that the comb generation process added minimal degradation to the baseline of the RF clock. These features, combined with its ease of use, have made the comb an integral part of our laboratory testing, and for this reason we believe it can become a workhorse in applications ranging from optical communication to arbitrary waveform generation. 


\section{REFERENCES}

[1] Th. Udem, R. Holzwarth, and T. W. Hänsch, "Optical frequency metrology", Nature, vol. 416, no. 6877, pp. 233-237, Mar. 2002.

[2] N. R. Newbury, "Searching for applications with a fine-tooth comb", Nature Photon., vol. 5, no. 12, pp. 853-857, Dec. 2010.

[3] D. J. Jones, S. A. Diddams, J. K. Ranka, A. Stentz, R. S. Windeler, J. L. Hall, and S. T. Cundiff, "Carrier-envelope phase control of femtosecond mode-locked lasers and direct optical frequency synthesis", Science, vol. 288, no. 5466, pp. 635-639, Apr. 2000.

[4] S. A. Diddams, D. J. Jones, J. Ye, S. T. Cundiff, J. L. Hall, J. K. Ranka, R. S. Windeler, R. Hozlwarth, Th. Udem, and T. W. Hänsch, "Direct link between microwave and optical frequencies with a $300 \mathrm{THz}$ femtosecond laser comb", Phys. Rev. Lett., vol. 84 , no. 22, pp. 5102-5105, May 2000.

[5] T. W. Hänsch, "Passion for precission", Rev. Mod. Phys., vol. 78, no. 4, pp. 1297-1309, Dec. 2006.

[6] A. Bartels, D. Heinecke, and S. A. Diddams, "10-GHz selfreferenced optical frequency comb", Science, vol. 326, no. 5953, pp. 681-681, Oct. 2009.

[7] A. D. Ellis and F. C. G. Gunning, "Spectral density enhancement using coherent WDM", IEEE Photon. Technol. Lett. vol. 17, no. 2, pp. 504-506, Feb. 2005.

[8] P. J. Delfyett, S. Gee, M. T. Choi, H. Izadpanah, W. Lee, S. Ozharar, F. Quinlan, and T. Yilmaz, "Optical frequency combs from semiconductor lasers and applications in ultrawideband signal processing and communications", J. Lightwave Technol. vol. 24 , no. 7 , pp. 2701-2719, Jul. 2006.

[9] D. Hillerkuss, R. Schmogrow, T. Schellinger, M. Jordan, M. Winter, G. Huber, T. Vallaitis, R. Bonk, P. Kleinow, F. Frey, M. Roeger, S. Koenig, A. Ludwig, A. Marculescu, J. Li, M. Hon, M. Dreschmann, J. Meyer, S. Ben Ezra, N. Narkiss, B. Nebendahl, F. Parmigiani, P. Petropoulos, B. Resan, A. Oehler, K. Weingarten, T. Ellermeyer, J. Lutz, M. Moeller, M. Huebner, J. Becker, C. Koos, W. Freude, and J. Leuthold, " $26 \mathrm{~Tb} / \mathrm{s}$ line-rate superchannel transmission utilizing all-optical fast Fourier transform processing", Nature Photon. Vol. 5, no. 6, pp. 364-371, Jun. 2011.

[10] C. B. Huang, D. E. Leaird, and A. M. Weiner, "Time-multiplexed photonically enabled radio-frequency arbitrary waveform generation with 100 ps transitions", Opt. Lett. vol. 32, no. 22, pp. 3242-3244, Nov. 2007.

[11] E. Hamidi, D. E. Leaird, and A. M. Weiner, "Tunable programmable microwave photonic filters based on an optical frequency comb", IEEE Trans. Microw. Theory Techn. vol. 58, no 11, pp. 3269-3278, Nov. 2010.

[12] V. R. Supradeepa, C. M. Long, R. Wu, F. Ferdous, E. Hamidi, D. E. Leaird, and A. M. Weiner, "Comb-based radiofrequency photonic filters with rapid tunability and high selectivity", Nature Photon. vol. 6, no. 3, pp. 186-194, Mar. 2012.

[13] M. H. Song, V. Torres-Company, A. J. Metcalf, and A. M. Weiner, "Multitap microwave photonic filters with programmable phase response via optical frequency comb shaping", Opt. Lett. vol. 37, no. 5, pp. 845-847, Mar. 2012.

[14] Z. Jiang, C. B. Huang, D. E. Leaird, and A. M. Weiner, "Optical arbitrary waveform processing of more than 100 spectral comb lines", Nature Photon. vol.1, no. 8, pp. 463-467, Aug. 2007.

[15] H. Murata, A. Morimoto, T. Kobayashi, and S. Yamamoto, "Optical pulse generation by electrooptic modulation method and its application to integrated ultrashort pulse generators", IEEE J. Sel. Top. In Quantum Electron., vol. 6, no. 6, pp. 1325-1331, Nov. 2000.

[16] T. Kobayashi, T. Sueta, Y. Matsuo, and Y. Cho, "High-repetitionrate optical pulse generator using a Fabry-Perot electrooptic modulator", Appl. Phys. Lett., vol. 21, no. 8, pp. 341-343, 1972.

[17] M. Kourogi, T. Enami, and M. Ohtsu, "A monolithic optical frequency comb generator", IEEE Photon. Technol. Lett. vol. 6, no. 2, pp. 214-217, Feb. 1994.

[18] T. Kobayashi, H. Yao, K. Amano, Y. Fukushima, A. Morimoto, and T. Sueta, "Optical pulse compression using high-frequency electro-optic phase modulation", IEEE J. Quantum Electron. vol. 24 , no. 2 , pp. $382-387$, Feb. 1988
[19] M. Fujiwara, J. Kani, H. Suzuki, K. Araya, and M. Teshima, "Flattened optical multicarrier generation of $12.5 \mathrm{GHz}$ spaced 256 channels based on sinusoidal amplitude and phase hybrid modulation", Electron. Lett. vol. 37, no. 15, pp. 967-968, Jul. 2001.

[20] T. Yamamoto, T. Komukai, K. Takada, and A. Suzuki, "Spectrally flattened phase-locked multi-carrier light generator with phase modulators and chirped fibre Bragg grating”, Electron. Lett. vol. 43, no. 19, pp. 1040-1042, Sep. 2007.

[21] T. Sakamoto, T. Kawanishi, and M. Izutsu, "Asymptotic formalism for ultraflat optical frequency comb generation using a Mach-Zehnder modulator", Opt. Lett. vol. 32, no. 11, pp. 15151517, Jun. 2007.

[22] I. L. Gheorma and G. K. Gopalakrishnan, "Flat frequency comb generation with an integrated dual-parallel modulator", IEEE Photon. Technol. Lett., vol. 19, no. 13, pp. 1011-1013, Jul. 2007.

[23] A. K. Mishra, R. Schmogrow, I. Tomkos, D. Hillerkus, C. Koos, W. Freude, and J. Leuthold, "Flexible RF-based comb generator", IEEE Photon. Technol. Lett. vol. 25, no. 7, pp. 701-704, Mar. 2013.

[24] S. Ozharar, F. Quinlan, I. Ozdur, S. Gee, and P. J. Delfyett, "Ultraflat optical comb generation by phase-only modulation of continuous-wave light", IEEE Photon. Technol. Lett. vol. 20, no. 1, pp. 36-38, Jan. 2008.

[25] R. Wu, V. R. Supradeepa, C. M. Long, D. E. Leaird, and A. M. Weiner, "Generation of very flat optical frequency combs from continous-wave lasers using cascaded intensity and phase modulators driven by tailored radio-frequency waveforms," Opt. Lett, vol. 35, no. 19, pp. 3234-3236, Oct. 2010.

[26] T. Otsuji, M. Yaita, T. Nagatsuma, and E. Sano, " $10-80 \mathrm{~Gb} / \mathrm{s}$ highly extinctive electro-optic pulse pattern generator" IEEE $J$. Sel. Top. In Quantum Electron. vol. 2, no 3, Sep. 1996.

[27] A. Ishizawa, T. Nishikawa, A. Mizutori, H. Takara, S. Aozasa, A. Mori, H. Nakano, A. Takada, and M. Koga, "Octave-spanning frequency comb generated by $250 \mathrm{fs}$ pulse train emitted from 25 $\mathrm{GHz}$ externally phase-modulated laser diode for carrier-envelopeoffset-locking", Electron. Lett. vol. 46, no. 19, pp. 1343-1344, Sep. 2010.

[28] A. Ishizawa, T. Nishikawa, A. Mizutori, H. Takara, H. Nakano, T. Sogawa, A. Takada, and M. Koga, "Generation of 120 -fs laser pulses at $1-\mathrm{GHz}$ repetition rate derived from continuous wave laser diode", Opt. Exp. vol. 19, no. 23, pp. 22402-22409, Oct. 2011.

[29] S. J. Xiao, L. Hollberg, N. R. Newbury, and S. A. Diddams, "Toward a low-jitter $10-\mathrm{GHz}$ source with an optical frequency comb generator”, Opt. Exp. vol. 16, no. 12, pp. 8498-8508, Jun. 2008.

[30] B. H. Kolner, "Space-Time duality and the theory of temporal imaging", IEEE J. Quantum Electron. vol. 30, no. 8, pp. 19511963, Aug. 1994.

[31] V. Torres-Company, J. Lancis, and P. Andres, "Space-Time analogies in optics", Prog. In Opt. vol. 56, pp. 1-80, 2011.

[32] J. vanHowe, J. Hansryd, and C. Xu, "Multiwavelength pulse generator using time-lens compression”, Opt. Lett. vol. 29, no. 13, pp. 1470-1472, Jul. 2004.

[33] J. Azana, "Time-to-frequency conversion using a single time lens", Opt. Commun. vol. 217, no. 1, pp. 205-209, Mar. 2003.

[34] V. Torres-Company, J. Lancis, and P. Andres, "Lossless equalization of frequency combs", Opt. Lett. vol. 33, no. 16, pp. 1822-1824, Aug. 2008.

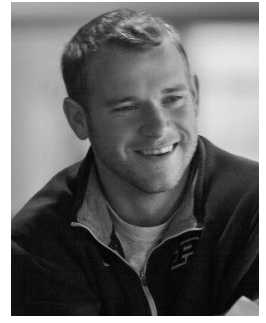

Andrew J. Metcalf was born in Madison, WI, in 1987. He received the B.S. degree (summa cum laude) in electrical engineering from the University of Wisconsin-Milwaukee in 2010, and the M.S.E.C.E degree from Purdue University in 2012. He is currently pursuing the Ph.D. degree in electrical engineering at Purdue. 
From 2008-2010 he worked as a co-op at Harley-Davidson Motor Company before becoming a Graduate Research Assistant in the Ultrafast Optics and Optical Fiber Communications group at Purdue. For his undergrad work, he received the coveted Deans Award for outstanding achievement in electrical and computer engineering. He is a member of both OSA and IEEE, an active reviewer, and has co-authored 9 journal and conference papers. His research interests include optical pulse shaping, frequency comb generation, and radio-frequency photonics.

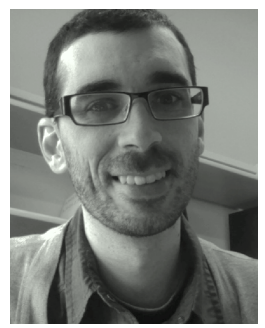

Victor Torres-Company is an Assistant Professor with the Microtechnology and Nanoscience department (MC2) at Chalmers University of Technology, Sweden. He obtained the PhD from the University of Valencia, Spain (2008). From 2009-2010 he was at the Photonics Systems group in McGill University, Canada, where he worked on ultrafast signal processing based on the space-time analogy. From 2010-2012 he was a Marie Curie IOF research fellow at the Ultrafast Optics group in Purdue University, USA. During his PhD, he spent several research stays at different European institutions, such as the Royal Institute of Technology (KTH), Sweden; the Technical University of Denmark (DTU); and the Institute of Photonic Sciences (ICFO) in Barcelona, Spain. For his $\mathrm{PhD}$ work, he won the Best Thesis award from the Applied Physics department at the University of Valencia. He is a member of the OSA and an active reviewer in OSA, IEEE, Elsevier and APS journals. He has co-authored more than 40 papers in leading optics journals. His main research interests include microwave photonics, optical frequency comb technology, silicon photonics, scalar coherence theory, and two-photon quantum interferometry.

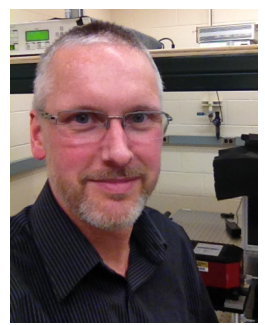

Daniel E. Leaird was born in Muncie, IN, in 1964. He received the B.S. degree in physics from Ball State University, Muncie, IN, in 1987, and the M.S. and Ph.D. degrees from the School of Electrical and Computer Engineering, Purdue University, West Lafayette, IN, in 1996 and 2000 respectively.

He joined Bell Communications Research (Bellcore), Red Bank, NJ, as a Senior Staff Technologist in 1987, and later advanced to Member of Technical Staff. From 1987 to 1994, he worked in the Ultrafast Optics and Optical Signal Processing Research Group, where he was a key team member in research projects in ultrafast optics, such as shaping of short optical pulses using liquid crystal modulator arrays, investigation of dark soliton propagation in optical fibers, impulsive stimulated Raman scattering in molecular crystals, and all-optical switching.

Dr. Leaird is currently a Senior Research Scientist and Laboratory Manager of the Ultrafast Optics and Optical Fiber Communications Laboratory in the School of Electrical and Computer Engineering, Purdue University, where he has been since 1994. He has co-authored approximately 100 journal articles, 150 conference proceedings, and has three issued U.S. patents.

$\mathrm{He}$ is active in the optics community and professional organizations including the Optical Society of America and IEEE Photonics Society where he served as the chair of the Ultrafast Optics technical committee from 2006-2009 as well as serving as a consultant to venture capitalists by performing technical due diligence. He also serves as a reviewer for Optics Letters, Optics Express, Photonics Technology Letters, Applied Optics, and Journal of the Optical Society of America $\mathrm{B}$ in addition to serving on National Science Foundation review panels in the SBIR program.

Dr. Leaird has received several awards for his work in the ultrafast optics field including a Purdue Professional Achievement Award, a Magoon Award for outstanding teaching, an Optical Society of America/New Focus Student Award, and a Bellcore "Award of Excellence".

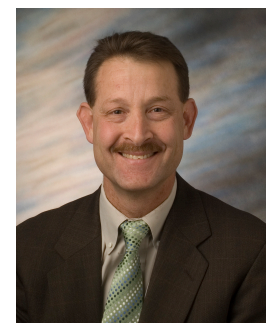

Andrew M. Weiner graduated from M.I.T. in 1984 with an Sc.D. in electrical engineering. Upon graduation he joined Bellcore, first as Member of Technical Staff and later as Manager of Ultrafast Optics and Optical Signal Processing Research. Prof. Weiner moved to Purdue University in 1992 and is currently the Scifres Family Distinguished Professor of Electrical and Computer Engineering. His research focuses on ultrafast optics signal processing and applications to high-speed optical communications and ultrawideband wireless. He is especially well known for his pioneering work on programmable femtosecond pulse shaping using liquid crystal modulator arrays. Prof. Weiner is author of a textbook entitled Ultrafast Optics and has published over 250 journal articles. He is a Fellow both of the OSA and of the IEEE and is a member of the U.S. National Academy of Engineering. He has won numerous awards for his research, including the Hertz Foundation Doctoral Thesis Prize, the OSA Adolph Lomb Medal, the ASEE Curtis McGraw Research Award, the International Commission on Optics Prize, the IEEE LEOS William Streifer Scientific Achievement Award, the Alexander von Humboldt Foundation Research Award for Senior U.S. Scientists, the OSA R.W. Wood Prize, and the IEEE Photonics Society Quantum Electronics Award. Prof. Weiner has served as Chair or Co-Chair of the Conference on Lasers and Electro-optics, the International Conference on Ultrafast Phenomena and the National Academy of Engineering's Frontiers of Engineering symposium, as Secretary/Treasurer of the IEEE Lasers and Electro-optics Society (LEOS), and as a Vice-President of the International Commission on Optics (ICO). He is currently Editor-in-chief of Optics Express. 